\title{
Procedures for Static Compaction of Test Sequences for Synchronous Sequential Circuits Based on Vector Restoration
}

\author{
Ruifeng Guo, Irith Pomeranz and Sudhakar M. Reddy ${ }^{+}$ \\ Electrical and Computer Engineering Department \\ University of Iowa \\ Iowa City, IA 52242
}

\begin{abstract}
We propose several compaction procedures for synchronous sequential circuits based on test vector restoration. Under a vector restoration procedure, all or most of the test vectors are first omitted from the test sequence. Test vectors are then restored one at a time or in subsequences only as necessary to restore the fault coverage of the original sequence. Techniques to speed-up the restoration process are investigated. These include limiting the test vectors initially omitted from the test sequence, consideration of several faults in parallel during restoration, and the use of a parallel fault simulator.
\end{abstract}

\section{Introduction}

It was shown in [1] that the length of a test sequence generated for a synchronous sequential circuit can be reduced in a postprocessing step that follows test generation, without losing fault coverage. One of the techniques proposed in [1] was based on test vector omission. During the vector omission procedure, test vectors are omitted from the test sequence one at a time or in subsequences. It was shown that the level of compaction achieved by this technique is significant for test sequences generated by various deterministic test generation procedures such as [2] and [3]. However, since the omission of each vector or subsequence requires resimulation of the test sequence to ensure that the fault coverage does not go down, the run time of the omission procedure from [1] is relatively high. Vector restoration was proposed in [4] as a more computationally efficient alternative to vector omission. Vector restoration based test compaction proceeds as follows. First, all or most of the test vectors are omitted from the test sequence. Test vectors are then restored one at a time or in subsequences only as necessary to restore the fault coverage of the original sequence. The reasons for preferring restoration over omission are the following.

(1) For many test sequences considered in [1] and [4], the test length after compaction is less than half of the original test length. This suggests that it may be faster to decide which test vectors must be restored into the test sequence in order to maintain the fault coverage, instead of deciding

+ Research supported in part by NSF Grant No. MIP-9220549, and NSF Grant No. MIP-9357581. on the test vectors that may be omitted.

(2) The effort required to decide whether a test vector needs to be restored is lower than the effort required to decide that a test vector can be omitted. To omit a vector, one must ensure that no detected fault becomes undetected after the omission. For this purpose, all the faults affected by the omission are simulated. To restore a test vector, it is sufficient to concentrate on one undetected fault, and restore test vectors until it is detected again.

The restoration based procedure of [4] showed significant run time advantages over the omission based procedure of [1]. However, the run times reported in [4] are still high. In this work, we investigate additional techniques to speed up the restoration based procedure, including the following.

(1) In [4], all the test vectors are initially omitted from the test sequence. Alternatively, the prefix of the test sequence that synchronizes the fault free circuit is retained, and the rest of the sequence is omitted. In this work, we use a heuristic to reduce the number of vectors that are initially omitted. This reduces the number of vectors that need to be considered for restoration, thus reducing the run time of the procedure.

(2) During the restoration process in [4], a single fault is considered at a time. In this work, we consider several faults in parallel during the restoration process. Faults are grouped together according to their detection time by the original test sequence. Thus, all the faults detected by the original test sequence at time unit $u_{i}$ are considered together for restoration of test vectors. Several heuristics are used to determine the order in which detection times will be considered. In addition, consideration of several detection times simultaneously reduces the computational effort significantly.

(3) An important characteristic of the proposed method is that existing parallel fault simulators can be used with a small amount of additional programming to implement the compaction procedure.

Other static compaction procedures for synchronous sequential circuits were recently described in [5] the [6]. The procedure of [5] uses repeated states, or states that appear twice or more along the test sequence, to find subsequences that can be omitted without reducing the fault coverage. Efficiency is often achieved in [5] at a signifi- 
cant loss in the ability to compact test sequences. The procedure of [6] is applicable to test sets comprised of multiple test subsequences. The procedure reorders the subsequences so as to shorten and/or eliminate some of them. A genetic optimization based procedure is used to determine an order that leads to an overall test length which is as small as possible.

The paper is organized as follows. In Section 2 we describe the necessary background. In Section 3 we present the new restoration based compaction procedures. Experimental results are given in Section 4. Section 5 concludes the paper.

\section{Preliminaries}

In this section, we provide the necessary background for the proposed procedures. We use the following notation.

The test sequence to be compacted is denoted by $T$. The length of $T$ is denoted by $L$. The test vector at time unit $u_{i}$ of $T$ is denoted by $t_{i}, 0 \leq i<L$.

The set of faults detected by $T$ is denoted by $F_{d e t}$. Fault simulation to determine $F_{\text {det }}$ is done using the conventional fault dropping approach. The set of faults detected by $T$ at time unit $u_{i}$ is denoted by $F_{d e t}(i)$. We denote by $u_{d e t}(f)$ the first detection time of a fault $f$ under the original test sequence $T$. Thus, if $u_{d e t}(f)=u_{i}$, then $f \in F_{\text {det }}(i)$.

The variable omitted indicates which test vectors are omitted from $T$. We have omitted $[i]=1$ if $t_{i}$ is omitted from $T$; otherwise, omitted $[i]=0$. For example, the test sequence $T=(00,01,10,11)$ from which $t_{1}$ was omitted is described by omitted $=(0,1,0,0)$. To simulate a test sequence with omitted vectors, we use the following rules. At time unit $u_{i}$, if omitted $[i]=0$, conventional simulation is carried out; if omitted $[i]=1$, simulation under $t_{i}$ is not required and the present state at time unit $u_{i+1}$ is equal to the present state at time unit $u_{i}$.

Next, we demonstrate the basic vector restoration procedure by considering ISCAS- 89 benchmark circuit $s 27$ under the test sequence shown in Table 1. Information about the faults detected by the sequence and their detection times are given in Table 2. For example, nine faults are detected at time unit 1 after the subsequence $\left(t_{0}, t_{1}\right)$ is applied.

The first two input vectors of the sequence take the fault free circuit from the all-unspecified initial state at time unit $u_{0}$ to state $0 x 0$ at time unit 1 , and to state 010 at time unit 2. Thus, the fault free circuit is synchronized at time unit 2. Since the synchronizing sequence of the fault free circuit is useful for the detection of all the faults, we retain it by setting omitted $[0]=0$ and omitted $[1]=0$. We omit the remaining vectors by setting omitted $[i]=1$ for $2 \leq i \leq 10$. The resulting sequence detects nine faults that are detected at time unit 1 by the original sequence (cf. Table 2). We consider the remaining faults starting with the ones detected by the original sequence at time unit 10 . For fault 10/0, we perform the following operations. We check whether the fault is detected by $T$ with the current vector omitted. The fault is not detected. We restore the vector at time unit 10 by setting omitted $[10]=0$, and resimulate the fault 10/0. The fault is now detected.

Table 1: A test sequence for $s 27$

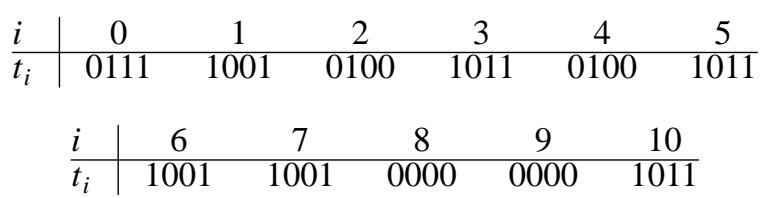

Table 2: Detection times for the sequence of $s 27$

\begin{tabular}{l|l}
$i$ & $F_{\text {det }}(i)$ \\
\hline 0 & $\phi$ \\
1 & $\{3 / 0,4 / 0,8 / 0,9 / 0,11 / 0,15 / 1$, \\
& $21 / 0,25 / 1,26 / 1\}$ \\
2 & $\{14 / 0,16 / 0,17 / 0,22 / 0,24 / 0\}$ \\
3 & $\{2 / 0,7 / 0,8 / 1,9 / 1,13 / 1,14 / 1$, \\
& $15 / 0,18 / 1,20 / 0,21 / 1,26 / 0\}$ \\
4 & $\phi$
\end{tabular}

\begin{tabular}{c|l}
$i$ & $F_{\text {det }}(i)$ \\
\hline 5 & $\phi$ \\
6 & $\{5 / 0,25 / 0\}$ \\
7 & $\phi$ \\
8 & $\phi$ \\
9 & $\{6 / 1,19 / 1,24 / 1\}$ \\
10 & $\{10 / 0,12 / 0\}$
\end{tabular}

Next, we consider the fault $12 / 0$, and perform the following operations. We check whether the fault is detected by $T$ with the current omitted vector. The fault is not detected. We restore the first omitted vector preceding the detection time of the fault. The detection time is 10 , and we have omitted $[10]=1$. The closest omitted vector is the one at time unit 9 . We set omitted [9] $=0$ and resimulate the fault $12 / 0$. The fault is still not detected. The fault $12 / 0$ is detected after omitted [8], omitted [7], omitted [6], omitted [5] and omitted [4] are all set to 0 .

We continue to consider faults detected by the original sequence at time unit 9 . The fault $6 / 1$ is such a fault. Simulating it using the current omitted vector, it turns out that the fault is detected. The same applies to all the other faults with detection times 9 and lower. The first pass over all the faults terminates with omitted $[2]=1$, omitted $[3]=1$ and omitted $[i]=0$ for $i \neq 2,3$.

Once all the faults in $F_{\text {det }}$ are considered and vectors are restored to detect each one of them, the resulting sequence is simulated again to ensure that no new faults become undetected. If a fault becomes undetected after considering other faults, additional vectors must be restored to detect it. This process is repeated until all the faults in $F_{d e t}$ are detected. We point out that this process will always terminate. In the worst case, it will terminate after restoring all the test vectors of the original sequence. In our example, all the faults are detected by the sequence with omitted $[2]=1$, omitted $[3]=1$ and omitted $[i]=0$ for $i \neq 2,3$. The resulting sequence is of length 9 , shorter by two vectors than the original sequence.

\section{Vector restoration based procedures}

In this section, we describe several restoration based compaction procedures. They differ from the procedure of [4] in the test vectors that are kept in the initial test sequence, and in the number of faults considered at every step. Specifically, consideration of several faults is achieved by grouping together faults that have the same detection time. 
In addition, the parallel fault simulator HOPE [7], [8] is used to perform all the fault simulations required during the compaction process. These changes contribute to speeding up the procedure significantly.

In all the procedures described in this section, the fault free initializing prefix of the test sequence is maintained as part of the compacted test sequence.

To facilitate the description of the procedures, we denote by $F_{\text {det }}^{\prime}$ the set of faults detected by the test sequence with a given omitted vector. Restoration is performed for faults in $F_{d e t}-F_{d e t}^{\prime}$, i.e., faults which are detected by the original test sequence but not detected with the current omitted vector. Once a vector or subsequence of vectors is restored, fault simulation may be carried out to update the set $F_{d e t}^{\prime}$.

\subsection{The first vector restoration based procedure}

Procedure 1 given in Figure 1 has the following new features.

We observe that when test vectors are restored to ensure the detection of a fault $f$ with $u_{\text {det }}(f)=u_{i}$, the first test vector to be restored is $t_{i}$. Thus, a test vector $t_{i}$ with $u_{d e t}(f)=u_{i}$ for $f \in F_{d e t}-F_{d e t}^{\prime}$ is likely to be included in the test sequence after restoration. Based on this observation, Procedure 1 leaves in the initial test sequence the following vectors. (1) The synchronizing prefix of the test sequence. This is done in Step 2 of Procedure 1. (2) Every test vector $t_{i}$ such that $u_{d e t}(f)=u_{i}$ for at least one fault $f$. This is done in Step 3 of Procedure 1.

For example, considering the test sequence given in Table 1 and the sets of detected faults given in Table 2, we set omitted $[0]=$ omitted $[1]=0$ due to the synchronizing prefix. In addition, we set omitted $[2]=$ omitted $[3]=$ omitted $[6]=$ omitted $[9]=$ omitted $[10]=0$ since at least one fault is detected in these time units.

For the faults in $F_{d e t}$ that remain undetected under the current omitted vector, we restore multiple test vectors in parallel, as follows. In Step 5(a) of Procedure 1, we identify the time units $u_{i_{1}}, u_{i_{2}}, \cdots, u_{i_{m}}$ such that there exists an undetected fault with detection time $u_{i_{j}}$ for $1 \leq j \leq m$. For each $u_{i_{j}}$, we identify the closest time unit $u_{k_{j}}$ which is not included in the test sequence $\left(u_{k_{j}} \leq u_{i_{j}}\right.$ and omitted $\left[k_{j}\right]=0$ ). We restore $t_{k_{j}}$ for $1 \leq j \leq m$. For example, consider the omitted vector shown in Table 3. Suppose that there is an undetected fault $f_{1}$ with $u_{\text {det }}\left(f_{1}\right)=u_{4}$, an undetected fault $f_{2}$ with $u_{\text {det }}\left(f_{2}\right)=u_{8}$ and an undetected fault $f_{3}$ with $u_{d e t}\left(f_{3}\right)=u_{9}$. In this case, we have $u_{i_{1}}=u_{4}, u_{i_{2}}=u_{8}$ and $u_{i_{3}}=u_{9}$. For $u_{i_{1}}=u_{4}$, the closest omitted vector is the one at time unit $u_{3}$. We thus have $u_{k_{1}}=3$. For $u_{i_{2}}=u_{8}$ and $u_{i_{3}}=u_{9}$, the closest omitted vector is the one at time unit $u_{7}$. We thus have $u_{k_{2}}=u_{k_{3}}=u_{7}$. In this case, we restore $t_{3}$ and $t_{7}$ simultaneously.

\section{Table 3: An example of Procedure 1}

\begin{tabular}{l|llllllllll}
$i$ & 0 & 1 & 2 & 3 & 4 & 5 & 6 & 7 & 8 & 9 \\
\hline omitted $[i]$ & 0 & 0 & 1 & 1 & 0 & 1 & 1 & 1 & 0 & 0
\end{tabular}

In Procedure 1, the set of detected faults and the detection times of all the faults are found in Step 1. The initial omitted vector is determined in Steps 2 and 3. The set $F_{d e t}^{\prime}$ of faults detected under the current omitted vector is computed in Step 4. Test vectors must be restored to detect the remaining faults, included in $F_{d e t}-F_{d e t}^{\prime}$. This is done in Step 5, where a single test vector preceding every detection time of a fault $f \in F_{\text {det }}-F_{d e t}^{\prime}$ is restored into the test sequence. Steps 4 and 5 are repeated as long as the omitted vector does not allow all the originally detected faults to be detected.

Procedure 1: Vector restoration based procedure 1

(1) Fault simulate $T$. Find the first detection time $u_{\text {det }}(f)$ of every fault $f$. Let $F_{d e t}$ be the set of detected faults (in our implementation, $F_{d e t}$ is the set of faults detected after the fault free synchronization prefix of the test sequence; faults detected during the prefix are detected by the compacted test sequence as well). Let $N_{\text {det }}$ be the number of faults in $F_{d e t}$. Let $S_{u_{i}}$ be the state of the fault free circuit at time unit $u_{i}$.

(2) Let $u_{\text {sync }}$ be the first time unit where $S_{u_{s y n c}}$ is fully specified. Set omitted $[i]=0$ for $0 \leq u_{i}<u_{\text {sync }}$ and omitted $[i]=1$ for $u_{i} \geq u_{\text {sync }}$.

(3) For every fault $f \in F_{\text {det }}$ : Let $u_{\text {det }}(f)=u_{i}$. Set omitted $[i]=0$.

(4) Find the faults in $F_{\text {det }}$ which are detected by $T$ with the current omitted vector. Let the set of detected faults be $F_{d e t}^{\prime}$. Let the number of faults in $F_{d e t}^{\prime}$ be $N_{d e t}^{\prime}$.

(5) If $N_{d e t}^{\prime}<N_{d e t}$ :

(a) For every time unit $u_{i}$, if there exists a fault $f \in F_{d e t}-F_{d e t}^{\prime}$ with $u_{d e t}(f)=u_{i}$, let $u_{k}$ be the highest time unit such that $u_{k} \leq u_{i}$ and omitted $[k]=1$.

(b) Set omitted $[k]=0$ for every time unit $u_{k}$ identified in Step 5(a).

(c) Go to Step 4 .

(6) Omit every test vector $t_{i}$ with omitted $[i]=1$, and stop.

\section{Figure 1: Procedure 1}

Once a compacted test sequence is obtained, Procedure 1 is applied again to omit additional test vectors. This process terminates when the last application of Procedure 1 does not reduce the test length further. We refer to the overall procedure as REST - OM0, where REST indicates that it is a restoration based procedure, and $O M 0$ indicates that the initial test sequence is obtained by omitting time units where no faults are detected.

\subsection{The second procedure}

Procedure 2 given in Figure 2 is different from Procedure 1 in the following ways.

Procedure 1 uses an initial omitted vector that keeps every test vector $t_{i}$ with $u_{d e t}(f)=u_{i}$ for some fault $f$. Compared to initially omitting all the test vectors, the advantage of Procedure 1 is that a smaller number of test vectors need to be considered for restoration. The disad- 
vantage is that a test vectors $t_{i}$ with $u_{d e t}(f)=u_{i}$ may not have to be maintained as part of a minimal length test sequence. In this case, the final test length produced by Procedure 1 may be higher than necessary. Procedure 2 alleviates this problem by including test vectors with $u_{\text {det }}(f)=u_{i}$ in the test sequence only when $f$ is one of the next target faults for restoration. This is done in Steps 6 to 9 , as follows. We denote by $N_{d e t}^{\prime}(i)$ the number of faults which are not detected by the test sequence with the current omitted vector, and for which $u_{d e t}(f)=u_{i}$. We randomly select a time unit $u_{i_{1}}$ with $N_{\text {det }}^{\prime}\left(i_{1}\right) \neq 0$. We repeat the selection to obtain $m$ time units $u_{i_{1}}, u_{i_{2}}, \cdots, u_{i_{m}}$ with $\sum_{j=1}^{m} N_{d e t}^{\prime}\left(i_{j}\right)$ as close as possible to a predetermined constant, 64 in our implementation. Thus, we obtain approximately 64 target faults. Only for these target faults we then restore test vectors in Step 10 of Procedure 2. This process is repeated until all the faults are detected again.

\section{Procedure 2: Vector restoration based procedure 2}

(1) Fault simulate $T$. Find the first detection time $u_{d e t}(f)$ of every fault $f$. Let $F_{d e t}$ be the set of detected faults. Let $S_{u_{i}}$ be the state of the fault free circuit at time unit $u_{i}$.

(2) Let $u_{\text {sync }}$ be the first time unit where $S_{u_{s y n c}}$ is fully specified. Set omitted $[i]=0$ for $0 \leq u_{i}<u_{\text {sync }}$ and omitted $[i]=1$ for $u_{i} \geq u_{\text {sync }}$.

(3) Unmark all the time units.

(4) Find the faults in $F_{\text {det }}$ which are detected by $T$ with the current omitted vector. Let the set of detected faults be $F_{d e t}^{\prime}$. Let the number of faults in $F_{d e t}^{\prime}$ be $N_{d e t}^{\prime}$.

(5) If $N_{\text {det }}=N_{d e t}^{\prime}$, omit every test vector $t_{i}$ with omitted $[i]=1$, and stop.

(6) For every time unit $u_{i}$, let $N_{d e t}^{\prime}(i)$ be the number of faults in $F_{d e t}-F_{d e t}^{\prime}$ with $u_{d e t}(f)=u_{i}$.

(7) If all the time units with $N_{d e t}^{\prime}(i) \neq 0$ are marked, unmark all the time units.

(8) Randomly select unmarked time units $u_{i_{1}}, u_{i_{2}}, \cdots, u_{i_{m}}$ such that $N_{d e t}^{\prime}\left(i_{j}\right) \neq 0$ for $1 \leq j \leq m$, until $\sum_{j=1}^{m} N_{d e t}^{\prime}\left(i_{j}\right)$ exceeds 64 for the first time or every time unit $u_{k}$ with $N_{d e t}^{\prime}(k) \neq 0$ is selected. Mark all the selected time units.

(9) Let $F_{\text {targ }}$ be the set of faults in $F_{\text {det }}-F_{\text {det }}^{\prime}$ which are detected at one of the selected time units.

(10) While $F_{\text {targ }} \neq \phi$ :

(a) For every selected time unit $u_{i_{j}}$, if there exists a fault $f \in F_{\text {targ }}$ with $u_{\text {det }}(f)=u_{i_{j}}$, let $u_{k_{j}}$ be the highest time unit such that $u_{k_{j}} \leq u_{i_{j}}$ and omitted $\left[k_{j}\right]=1$.

(b) Set omitted $\left[k_{j}\right]=0$ for every time unit $u_{k_{j}}$ identified in Step 10(a).

(c) Remove from $F_{\text {targ }}$ the faults which are detected by $T$ with the current omitted vector.

(11) Go to Step 4.

Figure 2: Procedure 2
Procedure 2 is called repeatedly with the new test sequence as input to omit additional test vectors, until the test length cannot be reduced any further. We refer to the overall procedure as $R E S T-R O 64$, where REST indicates that it is a restoration based procedure, $R O$ indicates that time units are considered in random order in Step 8, and 64 is the approximate number of faults targeted in every iteration of the procedure.

We also implemented another procedure, called Procedure 3, which is similar to Procedure 2, except that instead of randomly selecting the time units in Step 8, they are selected in reverse order starting from the highest detection time and proceeding towards the lower ones. When Procedure 3 is repeated on compacted test sequences until no further reduction in test length is possible, we call it Procedure REST - SO64.

\section{Experimental results}

In this section, we report on several experiments using Procedures 1, 2, 3 and their iterative versions REST - OM0, REST - RO64 and REST - SO64, respectively. The procedures were applied to test sequences generated by the test generation procedure STRATEGATE of [9]. These test generation procedures achieve high fault coverage for the benchmark circuits considered here. The STRATEGATE test sequences were provided to us by Dr. Michael Hsiao of Rutgers University, who also provided us the test lengths after the compaction procedure of [5] was applied to these sequences.

Table 4: Procedures 1, 2 and 3

\begin{tabular}{l|r|r|rr|rr|rr} 
& orig & \multicolumn{1}{|c|}{$[5]$} & \multicolumn{2}{|c|}{ Proc. 1 } & \multicolumn{2}{|c|}{ Proc. 2 } & \multicolumn{2}{|c}{ Proc. 3 } \\
circuit & \multicolumn{1}{c|}{ t.len } & t.len & t.len & time & t.len & time & t.len & time \\
\hline s298 & 194 & 179 & 124 & 0.4 & 124 & 0.4 & 119 & 0.3 \\
s344 & 86 & 79 & 66 & 0.2 & 66 & 0.2 & 60 & 0.2 \\
s382 & 1486 & 562 & 563 & 15.3 & 526 & 4.7 & 584 & 3.0 \\
s400 & 2424 & 740 & 708 & 21.4 & 695 & 6.8 & 851 & 7.9 \\
s444 & 1945 & 776 & 638 & 19.0 & 707 & 7.1 & 602 & 3.6 \\
s526 & 2642 & 1665 & 1337 & 37.1 & 1429 & 33.3 & 1399 & 18.3 \\
s641 & 166 & 132 & 119 & 0.3 & 118 & 0.3 & 109 & 0.2 \\
s713 & 176 & 143 & 138 & 0.5 & 130 & 0.4 & 129 & 0.3 \\
s820 & 590 & 396 & 558 & 2.3 & 515 & 4.4 & 500 & 2.4 \\
s832 & 701 & 411 & 615 & 3.2 & 581 & 8.0 & 542 & 4.1 \\
s1196 & 574 & 543 & 308 & 0.5 & 308 & 1.2 & 279 & 0.7 \\
s1238 & 625 & 564 & 323 & 0.7 & 320 & 1.3 & 293 & 0.8 \\
s1423 & 3943 & 2442 & 1338 & 307.0 & 1152 & 73.8 & 1116 & 40.0 \\
s1488 & 593 & 409 & 560 & 6.9 & 522 & 12.7 & 472 & 4.8 \\
s1494 & 540 & 415 & 502 & 8.8 & 508 & 14.2 & 469 & 6.2 \\
s5378 & 11481 & 10436 & 911 & 192.5 & 959 & 174.6 & 668 & 57.5 \\
s35932 & 257 & 217 & 182 & 204.3 & 178 & 310.1 & 144 & 193.8 \\
\hline am2910 & 2509 & 2180 & 466 & 41.8 & 472 & 15.6 & 449 & 10.0 \\
div16 & 1098 & 937 & 564 & 69.8 & 540 & 13.7 & 485 & 4.1 \\
mult16 & 1696 & 465 & 358 & 15.9 & 334 & 7.5 & 226 & 2.7 \\
pcont2 & 195 & 106 & 108 & 14.3 & 106 & 8.9 & 92 & 5.1 \\
piir80 & 417 & 316 & 320 & 84.7 & 325 & 175.5 & 254 & 37.9 \\
\hline total & 34338 & 24113 & 10806 & & 10615 & & 9842 &
\end{tabular}

In Table 4, we show the results obtained by Procedures 1, 2 and 3. After circuit name we show the test length of the original sequence. For comparison, we show 
the test length obtained after the compaction procedure of [5]. We then show the test length and run time of Procedures 1, 2 and 3, in this order. Run time is measured in seconds on an HP C180 workstation. It can be seen that for the majority of the circuits considered, all the proposed procedures achieve higher levels of compaction than the procedure of [5]. In several cases, Procedures 1, 2 and 3 achieve significantly higher levels of compaction than the procedure of [5], e.g., for circuits s1423, s5378 and am2910. The total test lengths given in the last row of each table are the sums of all the test lengths in the corresponding columns.

In Table 5, we show the results of Procedures REST-OM0, REST-RO64 and REST-SO64. The columns headed $S O 64+R O 64$ will be explained below. Comparing with the results of Table 4 , it can be seen that iterating over the compaction procedures helps reduce the test length, at the cost of increased run time. The investment of additional run time is justified in cases where a reduction in test length is necessary to allow the test sequence to fit in the tester memory.

Table 5: Procedures REST - OM0, RO64, SO64

\begin{tabular}{l|r|rr|rr|rr|rr} 
& orig & \multicolumn{2}{|c|}{ REST-OMO } & \multicolumn{2}{|c|}{ REST-RO64 } & \multicolumn{2}{|c|}{ REST-SO64 } & \multicolumn{2}{|c}{ SO64+RO64 } \\
circuit & t.len & t.len & time & t.len & time & t.len & time & t.len & time \\
\hline $\mathrm{s} 298$ & 194 & 106 & 2.2 & 108 & 1.6 & 104 & 0.8 & 97 & 1.3 \\
$\mathrm{~s} 344$ & 86 & 57 & 0.9 & 56 & 0.3 & 58 & 0.3 & 57 & 0.4 \\
$\mathrm{~s} 382$ & 1486 & 536 & 71.5 & 524 & 10.5 & 542 & 8.4 & 535 & 20.6 \\
$\mathrm{~s} 400$ & 2424 & 688 & 62.6 & 658 & 26.2 & 626 & 17.8 & 559 & 29.9 \\
$\mathrm{~s} 444$ & 1945 & 631 & 68.9 & 641 & 26.6 & 602 & 6.7 & 588 & 24.1 \\
$\mathrm{~s} 526$ & 2642 & 1325 & 299.2 & 1132 & 179.0 & 1097 & 61.6 & 1083 & 149.4 \\
$\mathrm{~s} 641$ & 166 & 113 & 1.0 & 105 & 1.0 & 101 & 0.5 & 97 & 0.9 \\
$\mathrm{~s} 713$ & 176 & 135 & 1.4 & 124 & 1.1 & 123 & 0.7 & 114 & 1.3 \\
$\mathrm{~s} 820$ & 590 & 539 & 7.4 & 413 & 27.6 & 458 & 9.3 & 400 & 24.6 \\
$\mathrm{~s} 832$ & 701 & 557 & 21.1 & 470 & 38.7 & 490 & 11.3 & 428 & 43.3 \\
$\mathrm{~s} 1196$ & 574 & 298 & 1.9 & 283 & 5.5 & 269 & 2.2 & 263 & 5.4 \\
$\mathrm{~s} 1238$ & 625 & 313 & 2.5 & 293 & 6.1 & 277 & 2.7 & 269 & 6.7 \\
$\mathrm{~s} 1423$ & 3943 & 932 & 1007.4 & 840 & 415.3 & 961 & 156.9 & 749 & 401.9 \\
$\mathrm{~s} 1488$ & 593 & 545 & 50.9 & 451 & 64.3 & 437 & 23.2 & 437 & 30.9 \\
$\mathrm{~s} 1494$ & 540 & 482 & 48.3 & 446 & 41.4 & 419 & 29.6 & 396 & 60.9 \\
$\mathrm{~s} 5378$ & 11481 & 665 & 491.4 & 597 & 919.7 & 560 & 126.2 & 558 & 253.3 \\
$\mathrm{~s} 35932$ & 257 & 168 & 836.1 & 173 & 1106.2 & 118 & 968.2 & 118 & 1121.0 \\
\hline am2910 & 2509 & 419 & 138.5 & 425 & 53.5 & 410 & 31.3 & 353 & 56.4 \\
div16 & 1098 & 548 & 341.5 & 481 & 56.3 & 470 & 20.1 & 455 & 68.2 \\
mult16 & 1696 & 239 & 44.1 & 232 & 27.1 & 171 & 6.7 & 154 & 14.9 \\
pcont2 & 195 & 89 & 35.3 & 85 & 28.3 & 60 & 15.2 & 60 & 17.6 \\
piir80 & 417 & 265 & 342.5 & 242 & 1799.0 & 230 & 164.8 & 229 & 339.2 \\
\hline total & 34338 & 9650 & & 8779 & & 8583 & & 7999 &
\end{tabular}

Procedures REST $-O M 0, \quad$ REST - RO64 and REST - SO64 terminate when an additional iteration does not reduce the test length any further. However, even when the test length saturates for a given procedure, it may be possible to further reduce the test length by using a different compaction procedure. To investigate this possibility, we applied Procedures REST - OM0, REST - RO64 and REST - SO64 in various orders. Overall, the shortest test sequences were obtained when we applied $R E S T-R O 64$ to the test sequences produced by REST - SO64. The results of this experiment are shown in the columns with heading SO64 + RO64 of Table 5. It can be seen that additional reductions in test length are possible by applying both procedures.

\section{Concluding remarks}

We proposed three compaction procedures for synchronous sequential circuits based on test vector restoration. New techniques were described to speed-up the restoration process. The first technique initially retained test vectors where faults are detected. The second technique consisted of consideration of several faults in parallel during restoration. This was achieved by considering all the faults with the same detection time together. A parallel fault simulator was used as part of the compaction procedures. Several orders of processing detection times were considered. The proposed procedures were combined to maximize the level of compaction that can be achieved.

In this work, we compacted test sequences given as single entities. By partitioning a test sequence into several subsequences or requiring the test generator to produce several subsequences, and compacting each subsequence separately with respect to its set of target faults, the compaction time can be reduced [10].

\section{References}

[1] I. Pomeranz and S. M. Reddy, "On Static Compaction of Test Sequences for Synchronous Sequential Circuits", 33rd Design Autom. Conf., June 1996, pp. 215-220.

[2] T. P. Kelsey and K. K. Saluja, "Fast Test Generation for Sequential Circuits", Intl. Conf. Comp. Aided Design, Nov. 1989, pp. 354-357.

[3] T. Niermann and J. H. Patel, "HITEC: A Test Generation Package for Sequential Circuits", European Design Autom. Conf., 1991, pp. 214-218.

[4] I. Pomeranz and S. M. Reddy, "Vector Restoration Based Static Compaction of Test Sequences for Synchronous Sequential Circuits", Intl. Conf. on Computer Design, Oct. 1997, pp. 360-365.

[5] M. S. Hsiao, E. M. Rudnick and J. H. Patel, "Fast Algorithms for Static Compaction of Sequential Circuit Test Vectors", VLSI Test Symp., April 1997, pp. 188-195.

[6] F. Corno, P. Prinetto, M. Rebaudengo and M. Sonza Reorda, "New Static Compaction Techniques of Test Sequences for Sequential Circuits", 1997 Europ. Design \& Test Conf., March 1997, pp. 37-43.

[7] H. K. Lee and D. S. Ha, "HOPE: An Efficient Parallel Fault Simulator for Synchronous Sequential Circuits", 1992 Design Autom. Conf., June 1992, pp. 336-340.

[8] H. K. Lee and D. S. Ha, "New Techniques for Improving Parallel Fault Simulation in Synchronous Sequential Circuits", 1993 Intl. Conf. on Computer-Aided Design, Oct. 1993, pp. 10-17.

[9] M. S. Hsiao, E. M. Rudnick, and J. H. Patel, "Sequential Circuit Test Generation Using Dynamic State Traversal", 1996 Europ. Design \& Test Conf., March 1996, pp. 22-28.

[10] I. Pomeranz and S. M. Reddy, "Partitioning of Test Sequences for Synchronous Sequential Circuits", Tech. Rep. 3-1-1997, ECE Dept., Univ. of Iowa. 